\title{
Nierenerkrankungen beim Kaninchen - Ursachen und Therapiemöglichkeiten
}

\author{
Barbara Glöckner
}

Kaninchen mit akuter oder chronischer Niereninsuffizienz werden in der Praxis häufig mit unspezifischen Symptomen vorgestellt. Neben der Allgemeinuntersuchung ist daher eine Blutuntersuchung zur Diagnosefindung unverzichtbar. Die „Basistherapie“ bei Nierenerkrankungen ist stets ähnlich, jedoch müssen je nach Ursache weitere Untersuchungen und ergänzende therapeutische Maßnahmen eingeleitet werden.

Einschränkungen der Nierenfunktion finden sich beim Kaninchen oft, sie treten jedoch nicht nur altersbedingt auf. Druckatrophien, die im Rahmen einer Nephrolithiasis entstehen, oder einige häufige Erkrankungen wie die Encephalitozoonose können auch bereits in jungem oder mittlerem Lebensalter zu einer Niereninsuffizienz führen.

Beim Kaninchen überwiegen chronische Nierenveränderungen, aber auch akute Niereninsuffizienzen kommen vor und sind als Notfall zu betrachten. Betroffene Kaninchen werden mit meist unspezifischen Symptomen wie reduzierter Futteraufnahme, Gewichtsverlust oder Mattigkeit vorgestellt. Im Rahmen einer ausführlichen Anamnese, gefolgt von einer gründlichen Allgemeinuntersuchung, müssen zunächst andere Ursachen für die Veränderungen ausgeschlossen werden. Die Diagnose kann dann mithilfe weiterführender Untersuchungsmethoden gestellt werden.

\section{Akute Niereninsuffizienz Ätiologie}

Die akute Niereninsuffizienz ist meist infektiös bedingt. Sie entwickelt sich in der Regel aus einer bakteriellen Nephritis. Trotz infektiöser Grundursache handelt es sich nahezu immer um eine Ein- zeltiererkrankung. In Ausnahmefällen kann auch eine vollständige Verlegung der Ureteren durch Konkremente die Ursache sein.

\section{Klinik}

Die akute Niereninsuffizienz geht mit unspezifischen Symptomen einher, die plötzlich auftreten. Betroffene Tiere erscheinen sehr matt bis somnolent und stellen die Futteraufnahme meist vollständig ein.

\section{Diagnose}

Die Allgemeinuntersuchung verbleibt bis auf die auffallende Teilnahmslosigkeit des Patienten und gelegentlich Palpationsschmerzen im Nierenbereich meist ohne besonderen Befund. Die fehlenden Befunde im Zusammenhang mit dem plötzlichen Auftreten der Apathie und Inappetenz sollten zumindest als Differenzialdiagnose an eine akute Niereninsuffizienz denken lassen, sodass Harnund Blutuntersuchungen zur weiteren Abklärung angezeigt sind.

In der chemischen Harnuntersuchung ist oft ein deutlich erniedrigter $\mathrm{pH}$-Wert festzustellen. Mikroskopisch lassen sich meist Leukozyten, Erythrozyten und Epithelien nachweisen. Der Urin sollte umgehend zur bakteriologischen Untersuchung eingeschickt werden.

Im Rahmen der Blutuntersuchung fallen neben einer Leukozytose massiv erhöhte Werte für Harnstoff und Kreatinin auf. Zusätzlich sind oft Veränderungen der Leberwerte festzustellen.

Sonografisch sind nicht grundsätzlich Veränderungen des Nierengewebes darstellbar.

\section{Therapie}

Eine intensive Infusionstherapie ( A Abb. 1, - Tab.1) und eine Breitbandantibiose müssen umgehend eingeleitet werden. Das Antibiotikum ist ggf. kurzfristig nach Antibiogramm umzustellen.

Trotz der eingeschränkten Stoffwechselleistung der Niere kann die Gabe eines Analgetikums (z.B. Metamizol) angezeigt sein, um das Allgemeinbefinden zu verbessern. Gleichzeitig muss mit einer bedarfsgerechten Zwangsfütterung z.B. Critical Care ${ }^{\circledR}$ (Albrecht), Rodicare Instant ${ }^{\circledR}$ (Alfavet), oder Herbi Care Plus (WDT) - begonnen werden.

Zur Unterstützung der Nierenfunktion haben sich zudem Präparate aus der homöopathischen Tiermedizin bewährt, z.B. SUC $^{\circledR}$ (Solidago comp. ad us. vet., Ubichinon comp., Coenzyme comp. ad us. vet., Heel) oder Renes/Viscum comp. PlantaVet. Die Präparate werden in der Akutphase $2 \times$ täglich eingesetzt und können subkutan oder oral appliziert werden.

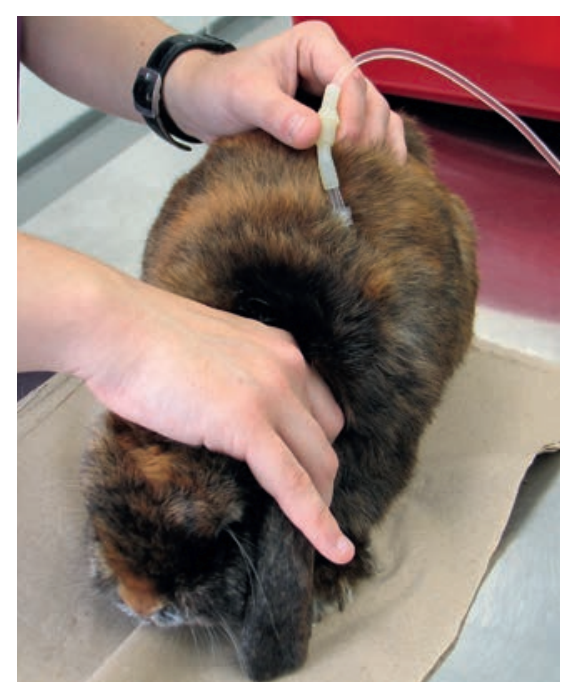

Abb. 1 Infusionen sind sowohl bei akuter als auch bei chronischer Niereninsuffizienz ein wichtiger Pfeiler der Therapie. Sie können je nach Zustand des Tieres s.c. oder i.v. verabreicht werden. 
Tab. 1 Therapie bei akuter Niereninsuffizienz.

\begin{tabular}{|c|c|c|}
\hline Maßnahme & Präparat & Dosierung \\
\hline $\begin{array}{l}\text { Infusionen mit } \\
\text { Vollelektrolytlösung }\end{array}$ & $\begin{array}{l}\text { z. B. Sterofundin }{ }^{\circledR} \text {, Ringer- } \\
\text { Lactat }^{\circledR}\end{array}$ & $\begin{array}{l}\text { - } 1 \times \text { tgl. } 80-100 \mathrm{ml} / \mathrm{kg} \text { i.v. oder } \\
\text { - } 2 \times \text { tgl. } 40 \mathrm{ml} / \mathrm{kg} \text { s. c. }\end{array}$ \\
\hline $\begin{array}{l}\text { Antibiotikum nach } \\
\text { Antibiogramm }\end{array}$ & $\begin{array}{l}\text { mit Breitbandantibiotikum } \\
\text { (z. B. Marbofloxacin) um- } \\
\text { gehend beginnen, ggf. } \\
\text { Wechsel nach Antibiogramm }\end{array}$ & - $1 \times$ tgl. $4 \mathrm{mg} / \mathrm{kg}$ s.c. \\
\hline ggf. Analgetikum & z. B. Metamizol (Novalgin ${ }^{\circledR}$ ) & $\begin{array}{l}3 \times \text { tgl. } 20-50 \mathrm{mg} / \mathrm{kg} \text { s. c. } \\
\text { oder p. } 0 .\end{array}$ \\
\hline \multirow[t]{2}{*}{$\begin{array}{l}\text { nierenunterstützende } \\
\text { homöopathische } \\
\text { Medikation }\end{array}$} & $\begin{array}{l}\text { SUC (Solidago comp. ad us. } \\
\text { vet., Ubichinon comp., } \\
\text { Coenzyme comp. ad us. vet.) }\end{array}$ & $\begin{array}{l}\text { initial } 2 \times \text { tgl. je } 0,5 \text { ml der } \\
\text { einzelnen Komponenten s. c. } \\
\text { oder p.o. für mindestens } \\
1 \text { Woche } \\
\text { ein Absenken oder Aus- } \\
\text { schleichen der Medikation } \\
\text { erfolgt individuell verlaufs- } \\
\text { abhängig }\end{array}$ \\
\hline & $\begin{array}{l}\text { Renes/Viscum comp. } \\
\text { PlantaVet }\end{array}$ & $\begin{array}{l}\text { - } 1-2 \times \text { tgl. } 1 \text { ml p. o. oder s.c. } \\
\text { Dauer der Gabe verlaufs- } \\
\text { abhängig }\end{array}$ \\
\hline Zwangsfütterung & $\begin{array}{l}\text { Critical Care }{ }^{\circledR} \text {, Rodicare } \\
\text { Instant }{ }^{\circledR} \text {, Herbi Care Plus }\end{array}$ & $\begin{array}{l}\text { ca. } 80 \mathrm{ml} / \mathrm{kg} / \mathrm{d} \text { auf mehrere } \\
\text { Mahlzeiten verteilt }\end{array}$ \\
\hline
\end{tabular}

\section{Prognose}

Aufgrund des rasanten Verlaufs ist die Prognose vorsichtig. Liegen Erregerresistenzen vor oder ist die Nierenfunktion irreversibel gestört, verschlechtert sich der Zustand des Tieres kontinuierlich weiter, sodass es euthanasiert werden sollte. Spricht der Patient jedoch gut auf die Infusionstherapie und die Antibiose an, sinken die Nierenwerte rasch und das Allgemeinbefinden stabilisiert sich. In diesem Fall ist eine dauerhafte Ausheilung möglich.

\section{Chronische Niereninsuffizienz Ätiologie}

Die chronische Niereninsuffizienz kommt beim Kaninchen im Gegensatz zur akuten Niereninsuffizienz oft vor. Häufige Ursachen sind:

- Encephalitozoonose (verursacht eine chronisch-interstitielle Nephritis)

- bakterielle chronische Pyelonephritis

- Konkremente in den Nieren und ableitenden Harnwegen

- altersbedingte Nierenfunktionseinschränkungen

- durch Neoplasien bedingte Veränderungen (z.B. Tumoren im Rahmen einer Leukose oder ein renales Karzinom).
Diese Möglichkeiten sind differenzialdiagnostisch zu berücksichtigen und können z.T. auch parallel auftreten.

\section{Klinik}

Kaninchen, die unter einer chronischen Niereninsuffizienz leiden, zeigen unspezifische Symptome wie schleichenden Gewichtsverlust und unterschiedlich stark ausgeprägte Inappetenz. Im weiteren Verlauf kommen struppiges Fell, blasse Schleimhäute, Exsikkose und Abmagerung hinzu.

\section{konkret}

Polydipsie und Polyurie können, müssen aber nicht vorhanden sein.

Sie sollten daher beim Kaninchen nicht als alleiniges Leitsymptom angesehen werden.

\section{Diagnose}

Die Anamnese und der Habitus des Patienten geben bereits erste Hinweise auf die Art der Erkrankung. Palpatorisch können ggf. je nach Grundursache Schmerzen im Nierenbereich oder auch vergrößerte Nieren festgestellt werden.
In der chemischen Harnuntersuchung mittels Teststreifen können eine Verschiebung des pH-Werts in den neutralen oder sauren Bereich sowie ein erhöhter Proteingehalt vorhanden sein. Im fortgeschrittenen Erkrankungsstadium sind auch Glukose und Keton nachweisbar. Der Urin wirkt sehr klar, da Kalziumkristalle nur noch in geringem Maße vorhanden sind. Während Erythrozyten und Leukozyten mikroskopisch nicht regelmäßig aufzufinden sind, lassen sich jedoch sehr häufig zahlreiche Epithelien nachweisen.

In der Blutuntersuchung sind Kreatinin und Harnstoff in variablem Ausmaß erhöht. Gleichzeitig kann Natrium erniedrigt und Kalium angestiegen sein. Häufig treten parallel milde bis ausgeprägte Anämien auf. Eine Leukozytose und Erhöhungen der Leberwerte als Ausdruck eines sekundären Infekts sind nicht regelmäßig festzustellen.

Vergrößerungen der Niere oder Nephrolithen bzw. Harnwegskonkremente sind bereits röntgenologisch sehr gut darstellbar. Im Rahmen einer Ultraschalluntersuchung sind zudem je nach Grundursache der chronischen Niereninsuffizienz dezente bis massive Veränderungen des Nierengewebes festzustellen.

\section{Therapie}

Die Therapie $(\vee$ Tab. 2 ) beginnt, wie auch bei der akuten Niereninsuffizienz, zunächst mit Infusionen. Diese können später oftmals auch vom Besitzer daheim regelmäßig verabreicht werden.

Zusätzlich können Präparate aus der homöopathischen/biologischen Tiermedizin vielfach helfen, die noch vorhandene Nierenfunktion aufrechtzuerhalten und zu unterstützen, sodass sich die Nierenwerte und das Allgemeinbefinden verbessern (z.B. SUC, Renes/Viscum comp. PlantaVet). Eine Erhöhung des Frischfutteranteils mit angepasstem Kalziumgehalt in der Rationszusammensetzung wirkt sich durch die Steigerung der Flüssigkeitsaufnahme ebenfalls langfristig positiv auf die Nierenfunktion aus. Gegebenenfalls muss eine Zwangsfütterung eingeleitet werden. Je nach Ursache und Ausprägung der Niereninsuffizienz muss 
Tab. 2 Allgemeine Therapie bei chronischer Niereninsuffizienz.

\begin{tabular}{|c|c|c|}
\hline Maßnahme & Präparat & Dosierung \\
\hline $\begin{array}{l}\text { Infusion mit Vollelek- } \\
\text { trolytlösung oder iso- } \\
\text { toner NaCl-Lösung (je } \\
\text { nach Elektrolythaushalt) }\end{array}$ & $\begin{array}{l}\text { z.B. Ringer-Lactat }{ }^{\circledR}, \\
\text { Sterofundin }{ }^{\circledR}, \mathrm{NaCl} \\
0,9 \%-\text { Braun }^{\circledR}\end{array}$ & $\begin{array}{l}1 \times \text { tgl. } 80-100 \text { ml/kg s. c. oder } \\
\text { i. v. } \\
\text { je nach Verlauf seltenere Appli- } \\
\text { kation (z.B. } 1-2 \times / \text { Woche) }\end{array}$ \\
\hline \multirow[t]{2}{*}{$\begin{array}{l}\text { nierenunterstützende } \\
\text { homöopathische } \\
\text { Medikation }\end{array}$} & $\begin{array}{l}\text { SUC (Solidago comp. } \\
\text { ad us. vet., Ubichinon } \\
\text { comp., Coenzyme } \\
\text { comp. ad us. vet.) }\end{array}$ & $\begin{array}{l}\text { initial je nach Höhe der Nieren- } \\
\text { werte } 1-2 \times \text { tgl. je } 0,5 \text { ml der } \\
\text { einzelnen Komponenten s.c. } \\
\text { oder p.o. für mindestens } \\
1 \text { Woche } \\
\text { ein Absenken oder Ausschlei- } \\
\text { chen der Medikation erfolgt } \\
\text { individuell verlaufsabhängig }\end{array}$ \\
\hline & $\begin{array}{l}\text { Renes/Viscum comp. } \\
\text { Planta Vet }\end{array}$ & - 1-2 × tgl. 1 ml p.o. oder s.c. \\
\hline $\begin{array}{l}\text { Optimierung der } \\
\text { Fütterung (hoher } \\
\text { Frischfutteranteil) }\end{array}$ & - & - \\
\hline ggf. Zwangsfütterung & $\begin{array}{l}\text { Critical Care }{ }^{\circledR} \text {, } \\
\text { Rodicare Instant }{ }^{\circledR} \text {, } \\
\text { Herbi Care Plus }\end{array}$ & $\begin{array}{l}\text { ca. } 80 \mathrm{ml} / \mathrm{kg} / \mathrm{d} \text { auf mehrere } \\
\text { Mahlzeiten verteilt bei voll- } \\
\text { ständiger Inappetenz }\end{array}$ \\
\hline
\end{tabular}

die Therapie symptomatisch oder kausal weiter ergänzt werden.

\section{Prognose}

Eine Regeneration des Nierengewebes und damit eine Ausheilung der chronischen Niereninsuffizienz ist nicht möglich. Das Ziel der Therapie ist daher, einen möglichst langen Zeitraum bei guter Lebensqualität zu erreichen. Ist dies aufgrund von erheblich erhöhten Nierenwerten oder einer ausgeprägten renalen Anämie im Zusammenhang mit deutlich eingeschränktem Allgemeinbefinden nicht realistisch zu erreichen, sollte mit den Besitzern über eine Euthanasie gesprochen werden. Gleiches gilt, wenn während der Therapie nicht bereits kurzfristig (etwa innerhalb von 2-3 Tagen) eine Stabilisierung oder Verbesserung des Befindens festgestellt werden kann. 
Sind von Beginn an nur milde Blutwertveränderungen festzustellen oder sinken die Werte in der ersten Therapiephase deutlich bei Verbesserung des Allgemeinbefindens, kann oftmals noch über eine Zeit von mehreren Monaten bis zu über 1 Jahr, in Ausnahmefällen auch länger, eine gute Lebensqualität aufrechterhalten werden.

Im Folgenden werden kurz die häufigsten Ursachen für eine chronische Niereninsuffizienz etwas genauer vorgestellt.

\section{Encephalitozoonose}

\section{Ätiologie}

Die Encephalitozoonose wird durch ein Protozoon, Encephalitozoon cuniculi, hervorgerufen. Der Erreger wird vorwiegend über den Urin übertragen. Ein hoher Prozentsatz der Heimtierkaninchen ist infiziert, jedoch erkrankt nur ein geringer Teil klinisch. Die Manifestation der Erkrankung scheint von verschiedenen Faktoren wie Stress und dem individuellen Immunstatus abhängig zu sein. Es ist daher nicht vorhersehbar, ob und wann ein Kaninchen im Laufe seines Lebens erkrankt.

\section{Klinik}

Neben den klassischen Verlaufsformen, die je nach Ausprägungsgrad mit Kopfschiefhaltung, Paresen oder weiteren neurologischen Ausfällen einhergehen, und der „Augenform“, die durch eine phakoklastische Uveitis gekennzeichnet ist, können auch die Nieren parallel oder isoliert betroffen sein. Es entwickelt sich eine chronische interstitielle granulomatöse Nephritis, die in ihrem Verlauf zu den Symptomen einer chronischen Niereninsuffizienz wie Gewichtsverlust und struppigem Fell führen kann.

\section{Diagnose}

Besteht aufgrund der klinischen Symptomatik der Verdacht auf eine Niereninsuffizienz, sollten eine Blut- und Harnprobe untersucht werden, um die Nierenfunktion zu überprüfen. Es ist jedoch nicht einfach, eine Encephalitozoonose eindeutig als Grundursache für eine chronische Niereninsuffizienz auszumachen. Die Blutuntersuchung ist hierbei nur bedingt hilfreich. Bei negativem serologischem Test kann die Encephalitozoonose sicher ausgeschlossen werden. Lassen sich jedoch Antikörper nachweisen, müssen trotzdem sämtliche Differenzialdiagnosen überprüft werden, um zu einer sicheren Diagnose zu gelangen.

\section{konkret}

Bei allen Tieren, die aufgrund einer neurologischen Form oder der Augenform der Encephalitozoonose vorgestellt werden, sollten die Nierenwerte überprüft werden.

Oft ist bereits eine Veränderung der Nierenwerte unterschiedlicher Ausprägung als Nebenbefund festzustellen.

\section{Therapie}

Sind gleichzeitig zentralnervöse Symptome/neurologische Ausfälle oder eine phakoklastische Uveitis vorhanden, sind diese zu behandeln. Ansonsten erfolgt die Therapie wie bei der chronischen Niereninsuffizienz. Parallel ist die Gabe von Fenbendazol $(1 \times$ tgl. $20-50 \mathrm{mg} / \mathrm{kg}$ p.o.) über mind. 3 Wochen ratsam, um die Erregerdichte zu verringern. Eine vollständige Erregerelimination ist nach derzeitigem Wissensstand leider nicht möglich. Bei neurologischen Ausfällen oder einer phakoklastischen Uveitis ist eine symptomatische Therapie einzuleiten.

\section{Prophylaxe/Metaphylaxe}

Derzeit wird diskutiert, Fenbendazol bei Encephalitozoonose-positiven Tieren routinemäßig in regelmäßigen Abständen (1/4- oder $1 / 2$-jährlich) einzusetzen. Die Medikation sollte jeweils über einen Zeitraum von 3 Wochen verabreicht werden. Ziel ist es, die Erregerdichte langfristig gering zu halten und damit unter anderem die Entstehung einer chronischen Niereninsuffizienz zu verzögern oder zu verhindern.

\section{Bei Encephalitozoonose-positiven Kaninchen ist es ratsam, den Flüssig- keitsanteil in der Nahrung durch ent- sprechende Zusammensetzung der Frischfutterration zu erhöhen, um die Nierenfunktion zu unterstützen.}

\section{Urolithiasis|Nephrolithiasis}

\section{Ätiologie}

Kaninchen sind aufgrund einer Stoffwechselbesonderheit prädisponiert, Konkremente in den Harnwegen zu bilden;

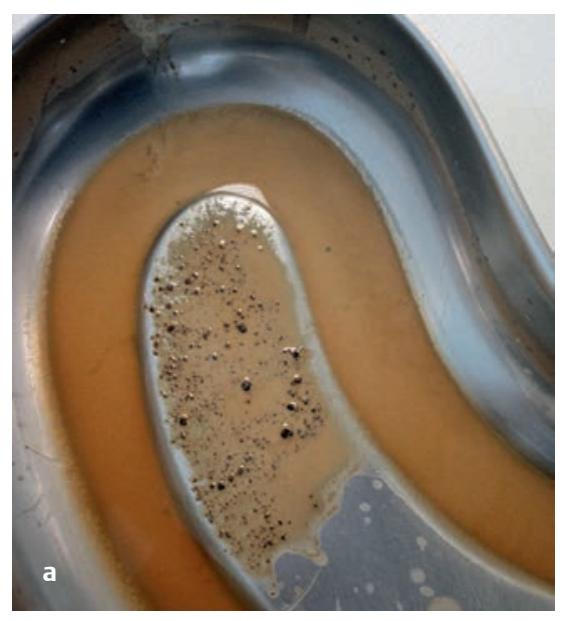

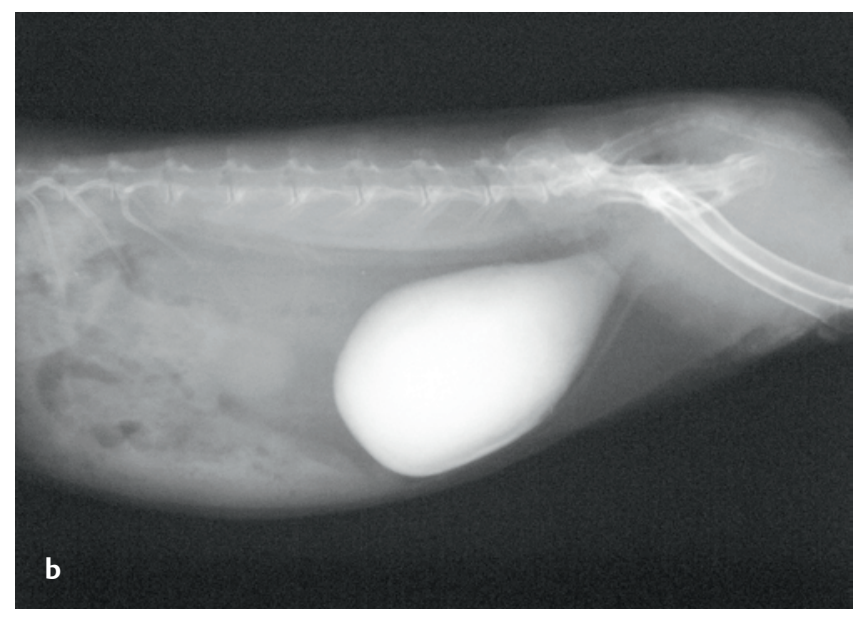

Abb. 2 Harngries. a) Harngries beim Kaninchen kann sehr feinsandig sein, aber auch grobe, scharfkantige Konkremente enthalten.

b) Kaninchen mit hochgradig griesgefüllter Harnblase. 
entsprechend häufig sind diese Veränderungen aufzufinden. Das Kaninchen resorbiert Kalzium nicht bedarfsgerecht, sondern nimmt es im Überschuss ins Blut auf, um ungenutzte Anteile über die Niere in den Urin zu filtern. Da der Harn-pH der Pflanzenfresser mit einem Wert zwischen 8 und 9 stark basisch ist, fällt Kalzium in diesem Milieu als Kristall aus. So weisen auch gesunde Kaninchen in der Regel einen getrübten Urin auf, der auf den Kalziumgehalt zurückzuführen ist.

Bei einer sehr kalziumbetonten Ernährung oder bei Flüssigkeitsmangel entstehen häufig Konkremente unterschiedlicher Größe ( Abb.2a) und Gestalt in der Blase oder Niere. Zusätzlich scheinen noch individuelle Prädispositionen und weitere Faktoren eine Rolle zu spielen.

Zu Schäden der Niere führen nicht nur Nephrolithen direkt, sondern gelegentlich auch Konkremente in den Ureteren, der Harnblase und der Urethra, da sie je nach Sitz und Größe zu einem Harnrückstau führen können.

\section{Klinik}

Die klinischen Symptome können insbesondere bei einer Urolithiasis, die durch mechanische Reizungen der Blasenwand eine Entzündung begünstigen kann, denen einer rein bakteriell bedingten Zystitis gleichen. Polyurie und Schmerzen beim Harnabsatz können daher im Vordergrund stehen. Liegt eine Nephrolithiasis vor, sind eher Symptome wie Mattigkeit und Gewichtsverlust vordergründig. Auch Schmerzanzeichen - wie eine zusammengekauerte Haltung mit gesträubtem Fell und halbgeschlossenen Augen - sind häufiger $z u$ beobachten. Polydipsie ist oft, aber nicht grundsätzlich festzustellen.

\section{Diagnose}

Im Rahmen der klinischen Untersuchung fällt bei einer Urolithiasis oftmals eine urinverschmierte Anogenitalregion mit teilweise stark geröteter und entzündeter Haut im Genitalbereich auf. Die Blase kann sich teigig-fest anfühlen (bei Blasengries) oder es kann ein Blasenstein zu palpieren sein. In der Harnuntersuchung sind meist Entzündungsanzeichen festzustellen.
Liegt eine Nephrolithiasis vor, bleibt die klinische Untersuchung oftmals bis auf eine Schmerzhaftigkeit im Nierenbereich oder bereits tastbar vergrößerte Nieren unauffällig.

Grundsätzlich sind im Verdachtsfall sowohl bei Urolithiasis ( $\triangle$ Abb. 3a) als auch Nephrolithiasis ( $\triangleright$ Abb.4) Röntgenaufnahmen in 2 Ebenen anzufertigen, um die Diagnose zu verifizieren. Je nach Sitz und Größe der Konkremente kann dann eine Therapieentscheidung getroffen und eine erste Prognose abgegeben werden. Die sonografische Untersuchung ist sinnvoll, um die Veränderungen des Nieren- gewebes und der ableitenden Harnwege beurteilen zu können. Blut- und Harnuntersuchungen liefern wichtige Informationen zur Einschränkung der Nierenfunktion.

\section{Therapie/Prophylaxe}

Neben der Therapie der chronischen Niereninsuffizienz und der begleitenden Harnwegsentzündung ist bei Blasensteinen eine chirurgische Entfernung meist unumgänglich. Liegt lediglich Blasengries vor, kann dieser ggf. durch vorsichtiges Ausmassieren der z.B. nach einer Infusion gut gefüllten Harnblase bei aufrechter Körperhaltung des Tieres ent- 

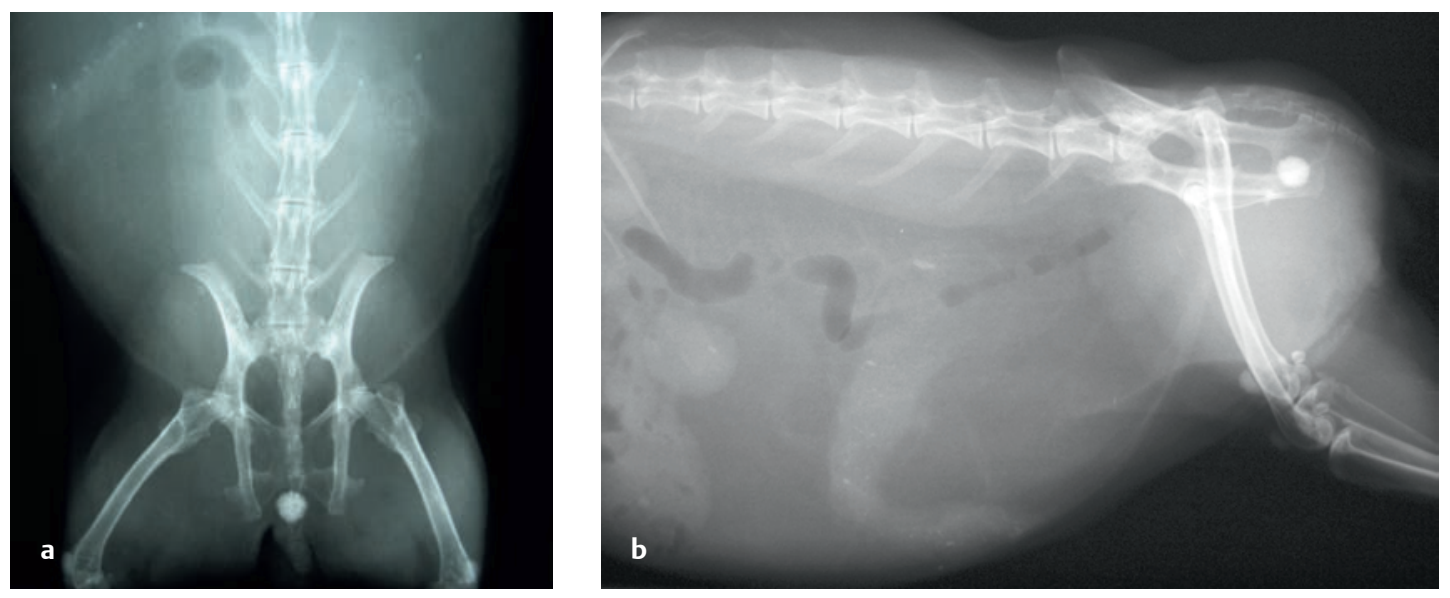

Abb. 3 Röntgenbilder eines Kaninchens mit Harnröhrenstein (weiterer Befund: Uterustumor). a) Ventrodorsale Ansicht.

b) Laterolaterale Ansicht.
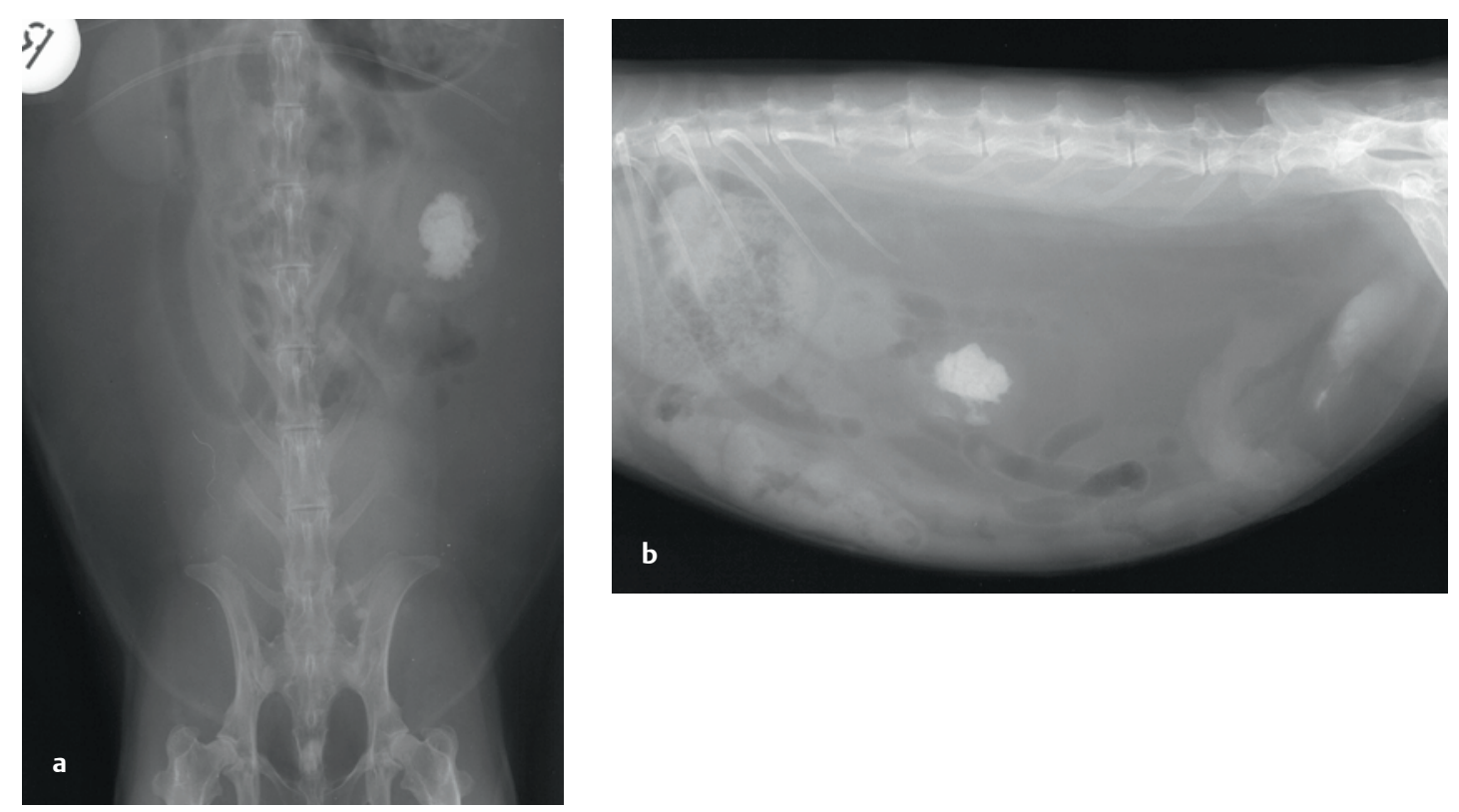

Abb. 4 Einseitige Nephrolithiasis (weiterer Befund: ggr. Blasengries, Uterustumor).

a) Ventrodorsale Ansicht. b) Laterolaterale Ansicht.

fernt werden. Ist dies nicht möglich, kann im Einzelfall eine Blasenspülung in Sedation erwogen werden.

Eine frischfutterbetonte und damit flüssigkeitsreiche Futterration ist therapeutisch und prophylaktisch von großer Bedeutung. Bei der Rationszusammenstellung muss darauf geachtet werden, kalziumärmer zu füttern bzw. kalziumreiche mit flüssigkeitsreichen Futtermitteln zu kombinieren. Kalziumhaltige Futtermittel dürfen jedoch nicht so radikal reduziert werden, dass der Körper Reserven mobilisieren muss und so in der Folge beispielsweise Zahnerkrankungen entstehen. Sogenannte Nagersteine sowie Luzernetrockenprodukte sollten jedoch grundsätzlich aus der Ration verbannt werden. Als weitere Prophylaxe- möglichkeit kann kalziumarmes Trinkwasser angeboten werden. Diese Fütterungsempfehlungen gelten auch für Kaninchen mit Nephrolithen, jedoch kann hierdurch lediglich das fortschreitende Wachstum der Nierensteine abgemildert werden.

Eine Therapie der Nephrolithiasis ist in der Regel nicht möglich. Eine einseitige Nephrektomie kann lediglich dann sinnvoll sein, wenn nur eine Niere Konkremente aufweist, die Nierenwerte im Normalbereich sind und die 2. Niere auch in der Sonografie ohne Befund ist. In allen anderen Fällen ist abhängig vom Allgemeinbefinden des Tieres lediglich die beschriebene unterstützende Behandlung möglich.

\section{konkret}

Eine Nephrektomie kommt nur infrage, wenn die röntgenologisch unauffällige Niere auch sonografisch ohne Befund ist und die Nierenwerte im Blut im Referenzbereich liegen.

Im Bedarfsfall sollte zusätzlich ein Analgetikum verabreicht werden, denn die Schmerzausschaltung ist ein wichtiger Faktor für die Lebensqualität. Da die Belastung der Niere zu berücksichtigen ist, ist dabei zwar stets eine ausreichend hohe, aber möglichst niedrige Dosis anzusetzen. Zudem kann ein homöopathisches Spasmolytikum (z.B. Spascupreel ${ }^{\circledR}$, Heel) eingesetzt werden, das problemlos vertragen wird. Wenn das Allgemeinbe- 

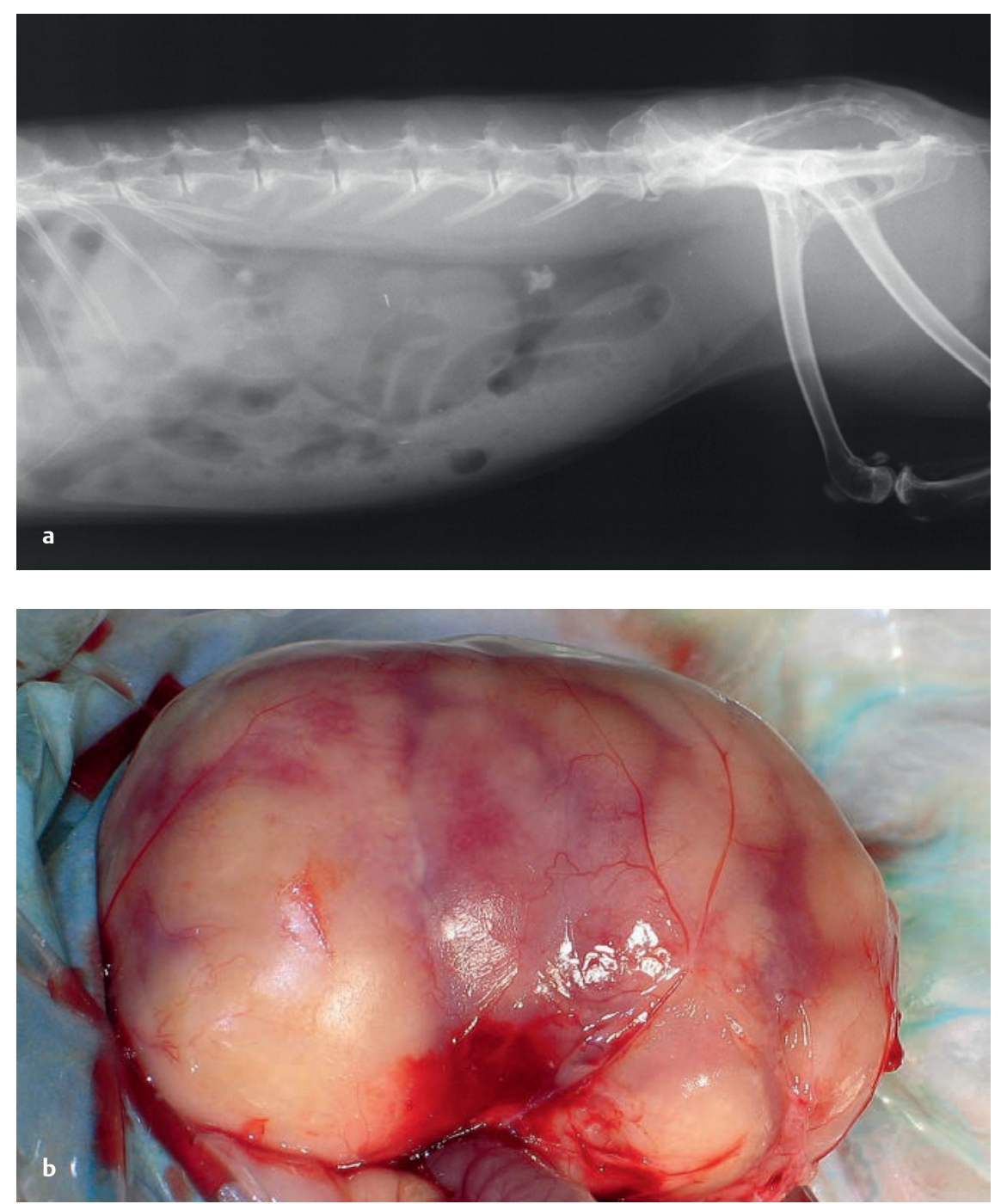

Abb. 5 Tumoröse Veränderungen der Niere im Rahmen einer Leukose.

finden des Tieres reduziert bleibt oder sich verschlechtert, sollte zur Euthanasie geraten werden.

\section{Tumoröse Veränderungen der Niere}

\section{Ätiologie}

Tumoröse Veränderungen der Niere $(\vee$ Abb.5) kommen beim Kaninchen nur gelegentlich vor. Dabei kann es sich um solitäre Tumoren wie das renale Karzinom, oder um viral bedingte Tumoren wie Lymphome im Rahmen einer Leukose handeln.

\section{Klinik}

Klinisch weisen die betroffenen Tiere nur unspezifische Symptome auf. Meist stehen die fortschreitende Abmagerung und Mattigkeit im Vordergrund. Bei solitären Tumoren ist in der Regel die Vergrößerung einer Niere gut zu palpieren. Im Rahmen der Leukose können in seltenen Fällen auch die Stammlymphknoten verdickt sein. Meist fallen aber lediglich Organschwellungen oder isolierte einoder beidseitige Nierenveränderungen auf.

\section{Diagnose}

Im Rahmen einer Ultraschalluntersuchung kann die Verdachtsdiagnose erhärtet werden. Im Falle eines renalen Karzinoms fällt in der Regel eine einseitige Masse auf, die vom ansonsten lediglich sekundär veränderten Nierengewebe abgrenzbar ist. Im Falle einer Leukose sind meist knotige Veränderungen $(\vee$ Abb.5b) einer oder beider Nieren sowie oftmals weiterer Organe (Milz, Leber) nachweisbar. 


\section{Therapieergänzungen bei chronischer Niereninsuffizienz}

Folgende Maßnahmen sind je nach Grundursache der chronischen Niereninsuffizienz ergänzend zur Basistherapie sinnvoll bzw. notwendig:

- Encephalitozoonose

- Fenbendazol ( $1 \times$ tgl. $20-50$ mg/kg p. o. über 3 Wochen)

- symptomatische Therapie bei neurologischen Ausfällen oder phakoklastischer Uveitis

- Urolithiasis

- Blasen- und Harnröhrensteine chirurgisch entfernen

- Blasenschlamm vorsichtig ausmassieren (ca. 1 Stunde nach Infusion) bzw. bedarfsweise Blasenspülung in Sedation

- Behandlung der Zystitis (Antibiose, Analgetikum)

- unterstützende Medikation, z. B. RodicareUro ${ }^{\circledR}$ (alfavet), allrodin ${ }^{\circledR}$ UTI Kn (almapharm)

- Nephrolithiasis

- Analgetikum

- bei einseitiger Nepholithiasis und vollständig intakter 2. Niere ggf. Nephrektomie

- Leukose

- keine Therapie möglich, Euthanasie bei Verschlechterung des Allgemeinbefindens

- solitäre Tumoren (z. B. renales Karzinom)

- bei vollständig intakter 2. Niere ggf. Nephrektomie

Zudem können ggf. entsprechende Verschiebungen der Zellfraktionen bzw. Veränderungen der Zellmorphologie im Blutausstrich festgestellt werden. Sind die Resultate nicht eindeutig, bringt eine Biopsie ein klares Ergebnis.

\section{Therapie/Prognose}

Liegt ein renales Karzinom vor, kann bei unveränderter 2.Niere und weitgehendem Ausschluss von Metastasen eine Nephrektomie durchgeführt werden. Die Prognose ist vorsichtig bis eher günstig zu bewerten. Bei der Leukose ist hingegen keine Therapie möglich, sodass die Prognose infaust ist. Betroffene Tiere sollten daher euthanasiert werden, sobald Einschränkungen des Allgemeinbefindens auffallen.
Alle Abbildungen: @ B. Glöckner

Online zu finden unter

http://dx.doi.org/10.1055/s-0035-1558505

\section{Literatur}

Literatur bei der Verfasserin.

\section{Dr. Barbara Glöckner}

c/o Tierarztpraxis Dr. Brieger

Anhaltiner Str. 2a

14163 Berlin 